\title{
Small-angle X-ray diffraction studies of a molluscan smooth muscle in the catch state
}

\author{
Yoshiko Tajima · Wataru Takahashi · \\ Akihiko Ito
}

Received: 19 October 2007 / Accepted: 1 July 2008/Published online: 18 July 2008

(C) The Author(s) 2008

\begin{abstract}
Small-angle X-ray diffraction patterns from the anterior byssus retractor muscle of Mytilus edulis in the resting, active, and catch states were examined closely to elucidate the structural features of catch. The specimens were isometrically contracted by stimulation with acetylcholine. The specimens that produced strong tensions in both the active and catch states showed noticeable structural change in the thick filaments. Although the tension was weaker in the catch state than in the active state, the axial spacings of the $14.5 \mathrm{~nm}$ meridional reflection and its higher order reflections from the thick filaments were more elongated in the catch state than in the active state. This means that the thick filaments were stretched more strongly in the catch state than in the active state.
\end{abstract}

Keywords Small-angle X-ray diffraction .

Meridional reflection - Molluscan smooth muscle .

Catch state $\cdot$ Thick filament

\section{Introduction}

The anterior byssus retractor muscle of Mytilus edulis (ABRM) is a myosin-regulated molluscan smooth muscle (Lehman et al. 1972) that produces a prolonged contraction called catch. In the catch state, a high but slowly decaying tension is maintained for a long time with very little expenditure of energy. To clarify the nature of the catch mechanism, researchers have attempted to detect structural changes in the catch state by X-ray diffraction techniques. Small-angle X-ray

Y. Tajima $(\varangle) \cdot$ W. Takahashi $\cdot$ A. Ito

Department of Physics, Tokyo Metropolitan University,

1-1 Minami-Ohsawa, Hachioji, Tokyo 192-0397, Japan

e-mail: ytajima@basil.ocn.ne.jp diffraction studies of the ABRM in the tonically contracting state, in which the tension level is due largely to catch tension, have shown an intensity increase of the $19 \mathrm{~nm}$ layer line from the actin-containing thin filaments (Lowy and Vibert 1972). The intensity increase was interpreted as a tropomyosin shift attributable to cross-bridge attachment to the thin filament. Time-resolved small-angle X-ray diffraction experiments have shown that the intensity increase of the $19 \mathrm{~nm}$ layer line from the relaxed to the catch state is much weaker than that seen during phasic contraction, and that it is close to the intensity increase in the rigor state (Popp et al. 1991).

Time-resolved small-angle X-ray diffraction studies of the equatorial reflection from the ABRM have revealed that the intensity of the $14 \mathrm{~nm}$ equatorial peak due to sideways packing of the thin filaments does not change when the ACh stimulation is terminated and that it remains almost constant for a while in the early stage of catch relaxation (Lowy and Poulsen 1982). This result was inferred by assuming that myosin heads remain attached to actin molecules in the rosette space around the thick filament. However, electron microscopic studies did not support that interpretation of the intensity change of the equatorial peak during contraction by insertion of myosin heads between the thin filaments arranged in rosettes (Bennett and Elliott 1989). Observation of the thin filament arrangement in cross sections of the freeze-substituted ABRM suggested that the intensity of the $14 \mathrm{~nm}$ equatorial peak comes mainly from the groups of thin filaments, not only in the resting state but also in the active and catch states.

According to medium-angle X-ray diffraction studies of the ABRM (Tajima et al. 1994a), the axial spacing of the $0.92 \mathrm{~nm}$ meridional reflection from the thin filaments increases in the active state and partially recovers in the catch state toward that in the resting state. On the other hand, the elongation of the axial spacing of the $1.038 \mathrm{~nm}$ 
meridional reflection from the thick filaments is maintained almost equally in the active and catch states. In our present work, the axial spacings and intensities of the thin and thick filament reflections in the small-angle X-ray diffraction pattern were measured more accurately by using an incident X-ray beam with improved convergence and longer camera lengths to clarify the structural changes of those filaments in the catch state. A brief report of this work was published previously (Tajima et al. 2001).

\section{Materials and method}

Anterior byssus retractor muscles about $30 \mathrm{~mm}$ long were dissected from large specimens of Mytilus edulis. The muscle was pared down to a bundle with a diameter of about $1 \mathrm{~mm}$ or less, and then mounted vertically in an acrylic specimen chamber with one end fixed in the chamber and the other attached to a force transducer. The distance between the ends of the muscle bundle was fixed

(a) $\mathbf{R}$

A

A

C

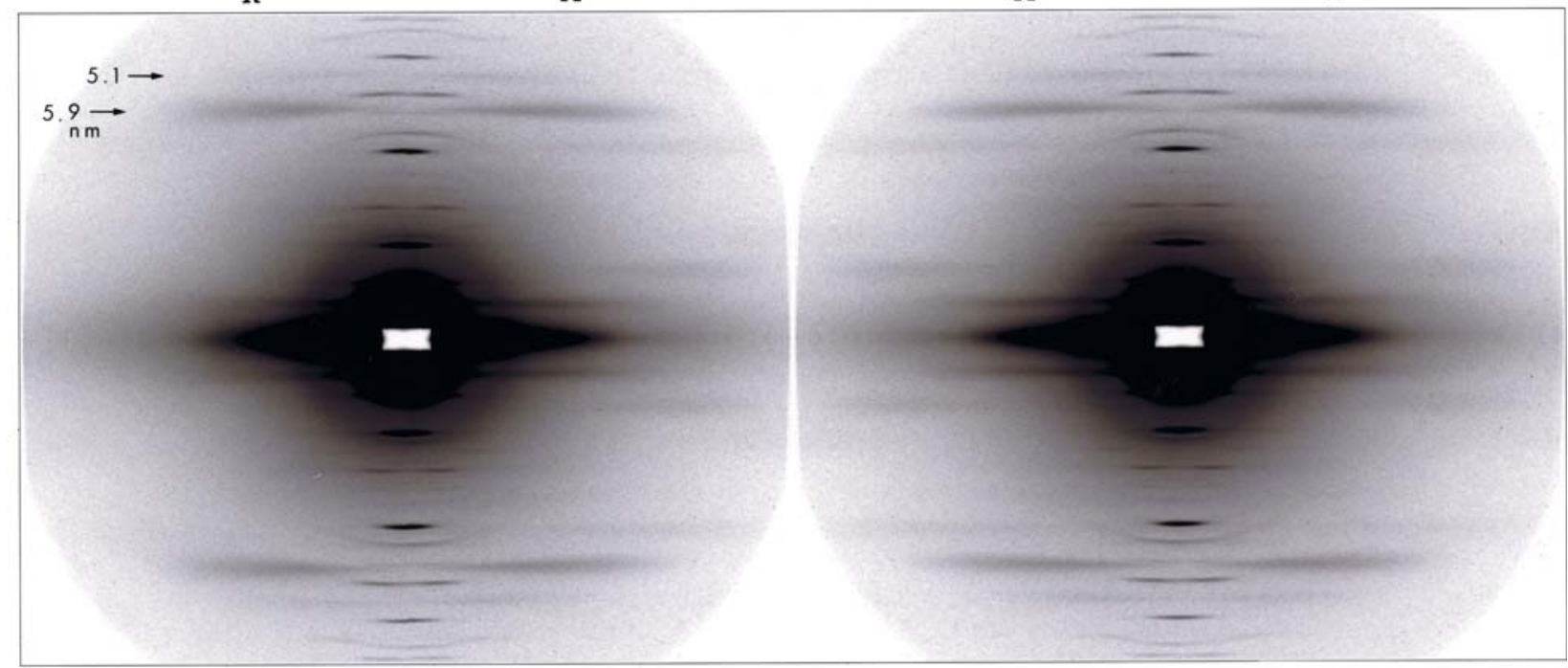

(b)

$\mathbf{R}$

A

A

C

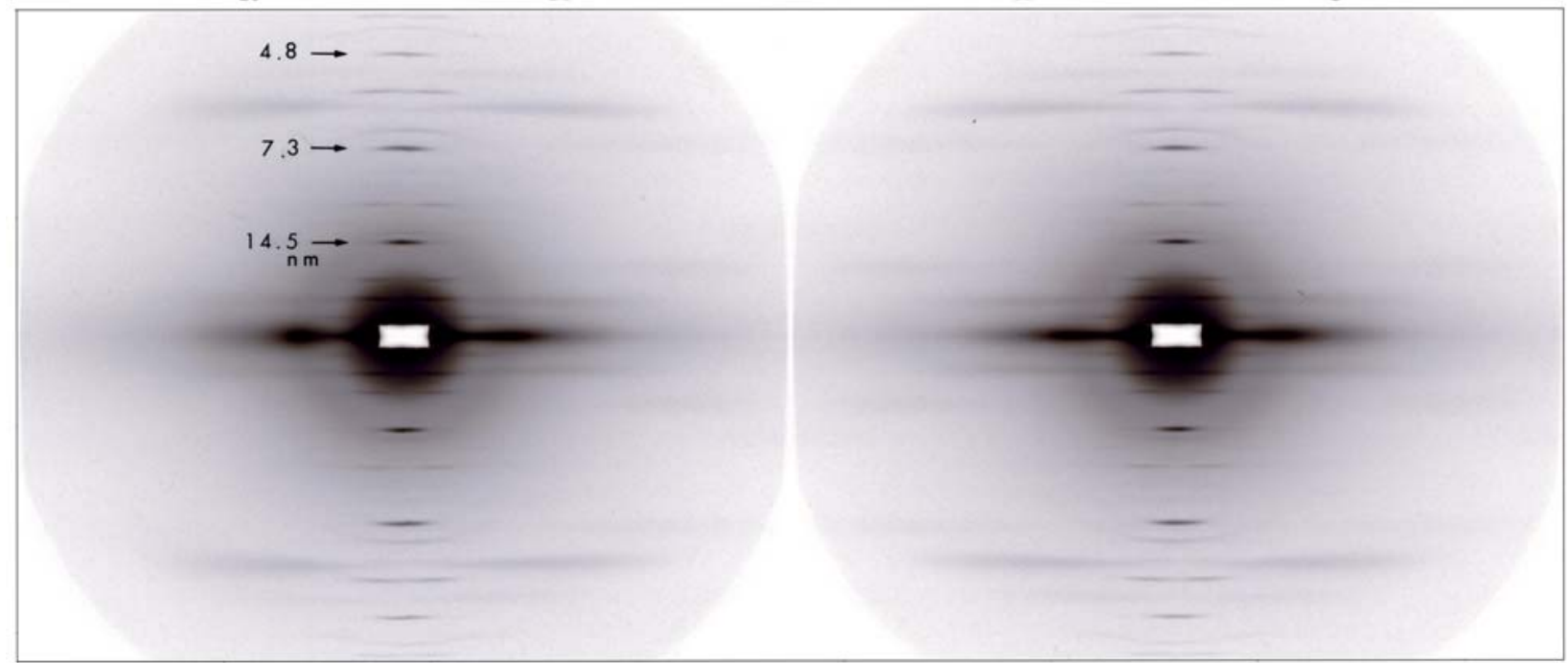

Fig. 1 Small-angle X-ray diffraction patterns from an ABRM compared between the resting (R), active (A), and catch (C) states. The X-ray patterns were recorded with a camera length of $2.2218 \mathrm{~m}$ at $13 \mathrm{~s}$ exposure. (a) and (b) show the same pattern, but (b) was reproduced with a gentle gradient to show the reflections close to the center of the X-ray pattern 
at a length beyond which the resting tension could be narrowly detected. The muscle bundle was maintained at $8^{\circ} \mathrm{C}$ in a flow of oxygenated artificial sea water through the specimen chamber, and contracted by stimulation with acetylcholine (ACh). In order to show the structural changes of the thin and thick filaments as distinctly as possible, we attempted to produce high tension using a high-concentration $\left(10^{-3} \mathrm{M}\right)$ ACh solution. The catch state was developed by washing out of ACh and terminated by applying 5-hydroxytryptamine $\left(2.5 \times 10^{-5} \mathrm{M}\right)$.

Small-angle X-ray diffraction experiments were carried out using synchrotron radiation and a point-focusing camera having the maximum camera length, $2 \mathrm{~m}$, at beamline 15A of the Photon Factory, Tsukuba. The X-ray patterns were recorded on $200 \times 200$ or $200 \times 250 \mathrm{~mm}^{2}$ imaging plates, and the patterns' digital intensity data were read from imaging plates with a pixel size of $0.1 \times 0.1 \mathrm{~mm}^{2}$. After the inclination angle and the center of each pattern were determined, the pattern was rotated so as to make the meridian parallel to the longitudinal axis of the pixel grid, and then translated so as to bring the center of the pattern to the nearest node of the pixel grid. Letting the node be the origin of the pixel grid, the four quadrants of the pattern were folded and averaged. For comparison of the X-ray patterns among the resting, active, and catch states, the intensity of each state was brought to the same scale by adjusting the background intensities to the same level at the area far from the center where the diffuse scattering from the muscle bundle, which was due primarily to the myosin heads, had diminished to almost zero (Poulsen and Lowy 1983; Lowy and Poulsen 1987).
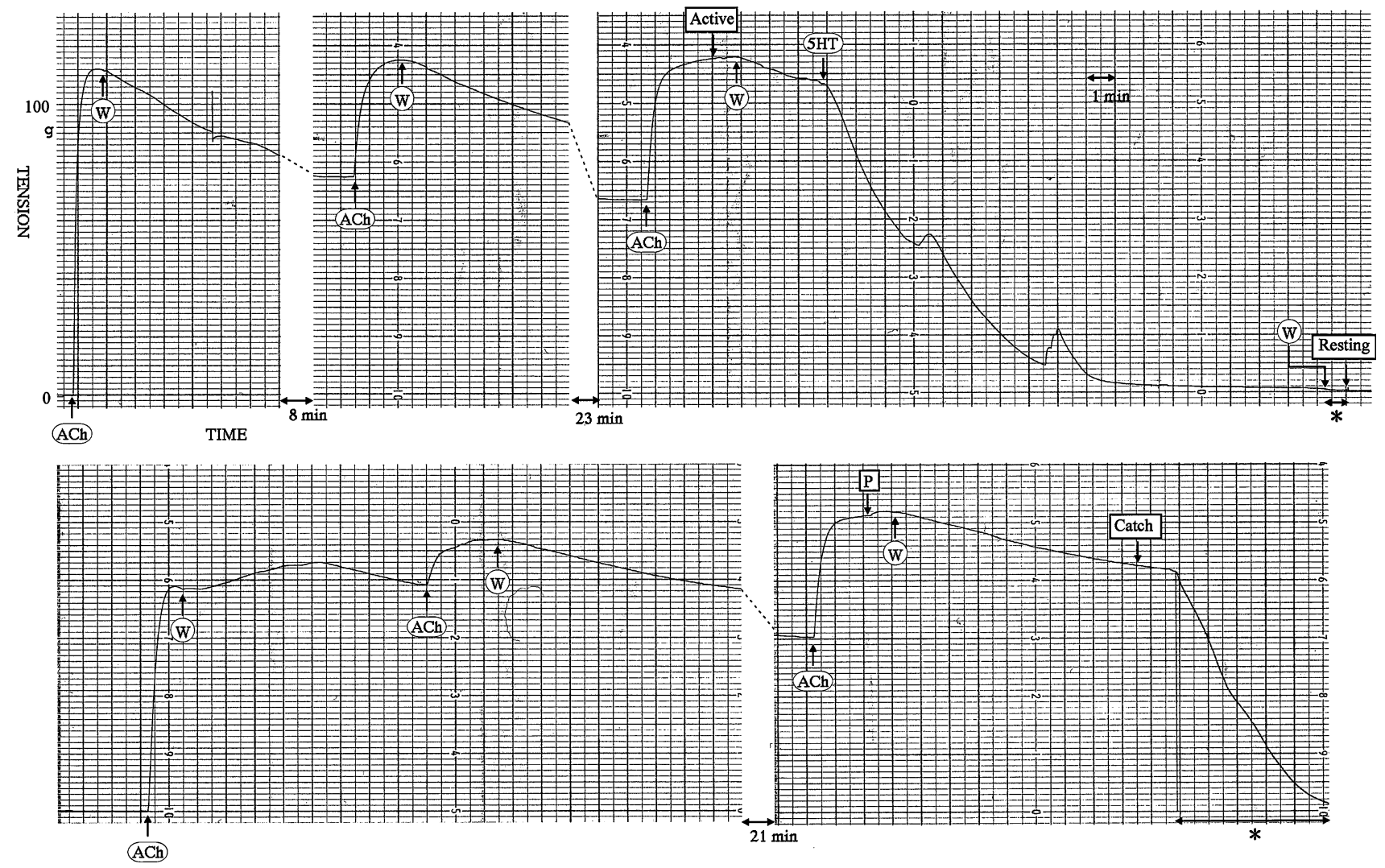

Fig. 2 Time dependence of the tension produced by the specimen while the small-angle X-ray diffraction patterns shown in Fig. 1 were recorded. Application of acetylcholine (ACh) and 5-hydroxytryptamine (5-HT), as well as washing out of them (W), are indicated by arrows. The asterisks show the intervals where the chart speed was slowed down by a factor of $1 / 60$. The $\mathrm{X}$-ray patterns in the active, resting, and catch states were recorded at the points indicated by the arrows. Point $\mathrm{P}$ shows the tension maximum in the active state just before the catch state in which the catch pattern was recorded. The strange behavior of the tension after the first stimulation in the lower panel was probably due to an irregular configurational change of the muscle. The muscle was so soft just after relaxation by $5-\mathrm{HT}$ that it be came elongated in the air by the force of gravity. During the exchange of the 5-HT solution for artificial sea water, a part of the muscle was probably caught at the lower edge of the narrow $(1 \mathrm{~mm})$ gap in the Xray window, and the tension produced by this part was not transmitted to the force transducer at the beginning. With the increase in tension, the irregular configuration gradually recovered, and simultaneously the tension recovered. The tension changed normally after the second and third stimulations, and it was ascertained by looking through a telescope after the second stimulation that the muscle was normal in its configuration and position in the cell. This irregularity was brought about probably due to the relatively large diameter of the specimen. Such a strange change in tension was not observed in the other specimens 


\section{Results}

Meridional reflections from thick filaments

Small-angle X-ray diffraction patterns from the same specimen in the resting, active, and catch states are compared in Fig. 1. Figure 2 shows the development of tension during the experiment to record the X-ray patterns shown in Fig. 1. The pattern of the catch state was taken 8 min after the end of the stimulation, when the tension was decaying very slowly. The $\mathrm{X}$-ray patterns were recorded on an imaging plate mounted inside an evacuated beam path. Because it took $10 \mathrm{~min}$ to evacuate the path after the imaging plate was mounted, it was difficult to take X-ray patterns in successive active and catch states. Therefore, the specimen was relaxed after the pattern in the active state was taken, and contracted again to record the pattern in the catch state. The tension was maintained at 12.2 and $8.8 \mathrm{~kg} / \mathrm{cm}^{2}$ during X-ray exposure in the active and catch states, respectively. The maximum tension in the active state just before the catch state in which the catch pattern was recorded was $10.7 \mathrm{~kg} / \mathrm{cm}^{2}$ (point $P$ in Fig. 2). The specimens that produced such strong tensions showed noticeable changes in the axial spacings of the thick filament reflections.
Figure 3 shows the radially integrated intensities of the three meridional reflections from the thick filaments corresponding to the $14.5,7.3$, and $4.8 \mathrm{~nm}$ axial spacings (Fig. 1b). Table 1 shows the axial spacings of the three reflections obtained from the positions of the maximum points on the fitting curves. Despite the decrease in tension, the axial spacings were more elongated in the catch state than in the active state.

The axial spacings in the catch state were elongated in the specimens that developed tensions stronger than $11 \mathrm{~kg} /$ $\mathrm{cm}^{2}$ in the active state. The tensile strength depended on the specimen; such strong tension occurred in $30 \%$ of the experiments. The elongation ratios of the spacings in the active and catch states are shown in Table 2 for three cases. Figure 1 shows the X-ray patterns of one of those cases, which is specimen (1) in Table 2. In all three cases, the axial spacings were longer in the catch state than in the active state. In these cases, the catch tension exceeded $70 \%$ of the tension in the active state, and tension decay after the end of the stimulation was less than $20 \%$.

Because of the decrease in tension, the three axial spacings will be smaller at point $\mathrm{P}$ in Fig. 2 than at the point where the pattern in the active state was recorded.
Fig. 3 Background-subtracted intensity distributions obtained by radial integration within the region -0.046 to $0.046 \mathrm{~nm}^{-1}$, which contained the meridional reflections from the thick filaments. The intensity distributions of the (a) 14.5, (b) 7.3 , and (c) $4.8 \mathrm{~nm}$ peaks are compared among the resting, active, and catch states. Circle: Observed intensity. Solid line: Fitting curve expressed by the pseudo-Voigt function, which is given by the sum of a Gaussian and a Cauchy function in an arbitrary proportion
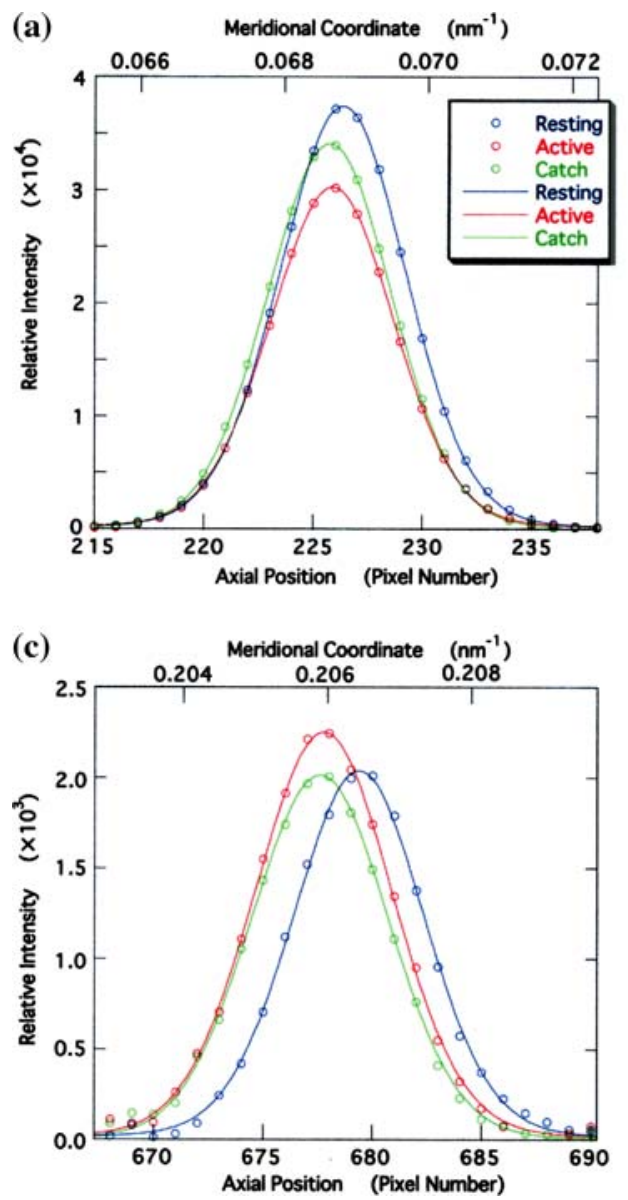

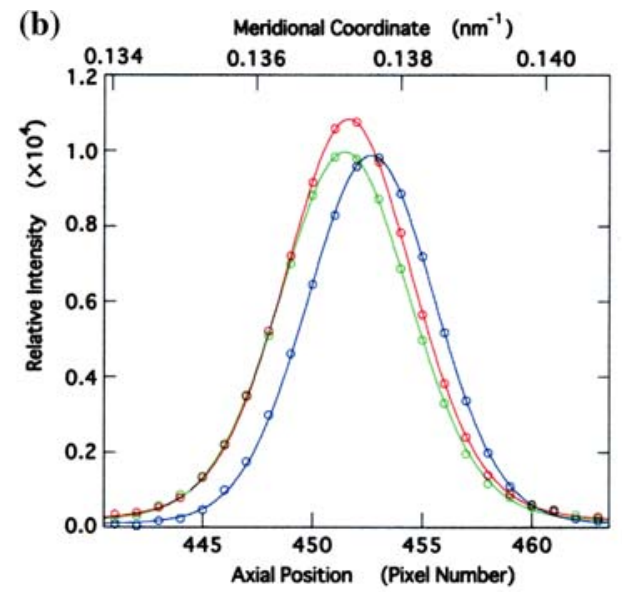


Table 1 Axial spacings and changes in integrated intensities of the meridional reflections from the thick filaments

\begin{tabular}{llll}
\hline State & $\begin{array}{l}\text { Position }^{\mathrm{a}} \\
\text { (Pixel number) }\end{array}$ & $\begin{array}{l}\text { Spacing } \\
(\mathrm{nm})\end{array}$ & $\begin{array}{l}\text { Intensity change }^{\mathrm{b}} \\
(\%)\end{array}$ \\
\hline $14.5 \mathrm{~nm}$ & & & \\
Resting & 226.351 & $14.533^{\mathrm{c}}$ & 0 \\
Active & 225.875 & 14.564 & -20.34 \\
Catch & 225.750 & 14.572 & -11.10 \\
$7.3 \mathrm{~nm}$ & & & \\
Resting & 452.670 & 7.268 & 0 \\
Active & 451.640 & 7.285 & 17.82 \\
Catch & 451.460 & 7.288 & 8.45 \\
$4.8 \mathrm{~nm}$ & & & \\
Resting & 679.373 & 4.844 & 0 \\
Active & 677.760 & 4.856 & 15.67 \\
Catch & 677.600 & 4.857 & 0.70 \\
\hline
\end{tabular}

a The position of the maximum point on the fitting curve shown in Fig. 3

b Change in the intensity obtained by integration of the area below the fitting curve

c The wavelength $0.14805 \mathrm{~nm}$ was used for the calculation of axial spacings. It was obtained by putting the average structural period of the thick filaments, as obtained from the three meridional reflections from the resting ABRM, into the period that had been measured with high accuracy by a conventional X-ray apparatus using $\mathrm{CuK} \alpha 1$ radiation (Tajima et al. 1994a)

Table 2 Increases in the axial spacings of the meridional reflections from the thick filaments in the active and catch states

\begin{tabular}{|c|c|c|c|c|c|c|}
\hline \multirow[t]{3}{*}{ Spacing } & \multicolumn{6}{|c|}{ Specimen } \\
\hline & \multicolumn{2}{|l|}{ (1) } & \multicolumn{2}{|l|}{ (2) } & \multicolumn{2}{|l|}{ (3) } \\
\hline & Active & Catch & Active & Catch & Active & Catch \\
\hline $14.5 \mathrm{~nm}$ & $0.21 \%$ & 0.27 & 0.21 & 0.26 & 0.33 & 0.37 \\
\hline 7.3 & 0.23 & 0.27 & 0.22 & 0.24 & 0.34 & 0.36 \\
\hline 4.8 & 0.24 & 0.26 & 0.22 & 0.24 & 0.35 & 0.38 \\
\hline
\end{tabular}

Therefore, it was supposed that the axial spacings would increase as the specimen changed from the active to the catch state. This supposition was tested by using a beam path that was altered to allow external mounting of the imaging plate. By using this beam path, small-angle X-ray diffraction patterns in the active and catch states were recorded successively without the need to relax the specimen between states (Fig. 4). Two higher-order meridional reflections from the thick filaments were recorded by decreasing the camera length slightly. Figure 5 shows the tension record through the recording of the patterns shown in Fig. 4. The tension was maintained at 12.0 and $10.4 \mathrm{~kg} /$ $\mathrm{cm}^{2}$ during X-ray exposure in the active and catch states, respectively. The radially integrated intensities of the five meridional reflections from the thick filaments showed that

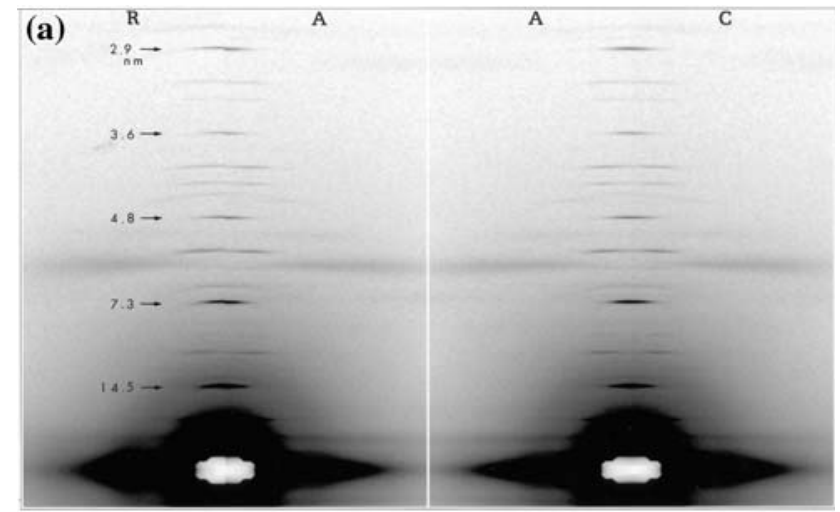

(b)

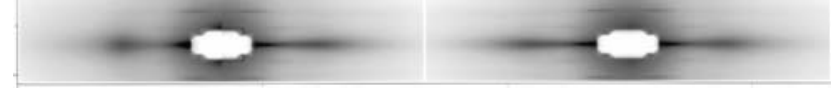

Fig. 4 Upper halves of the small-angle X-ray diffraction patterns from an ABRM compared between the resting (R), active (A), and catch $(\mathrm{C})$ states. The patterns were recorded with a camera length of $1.6490 \mathrm{~m}$ at $13 \mathrm{~s}$ exposure. (b) shows the reflections close to the centers of the patterns shown in (a) by reproduction with a gentle gradient

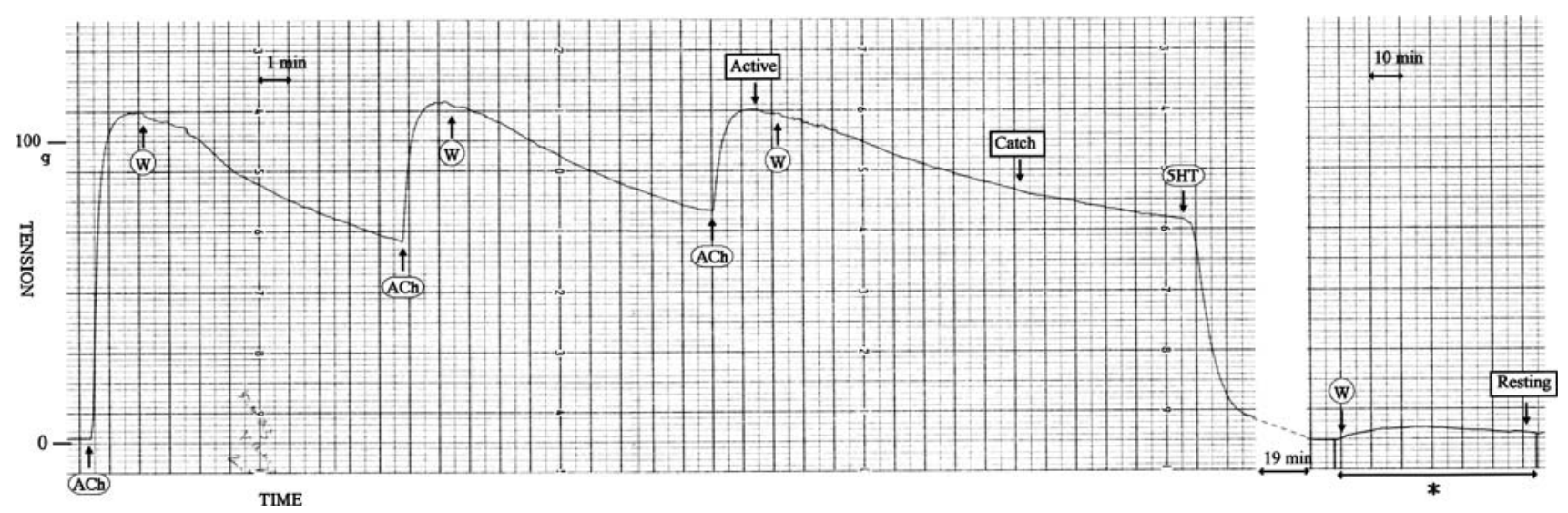

Fig. 5 Time dependence of the tension produced by the specimen while the small-angle X-ray diffraction patterns shown in Fig. 4 were recorded 
Fig. 6 Background-subtracted intensity distributions of the (a) 14.5 , (b) 7.3 , (c) 4.8 , (d) 3.6, and (e) $2.9 \mathrm{~nm}$ meridional reflections obtained by radial integration. The intensity distributions are compared among the resting, active, and catch states. Circle: Observed intensity. Solid line: Fitting curve
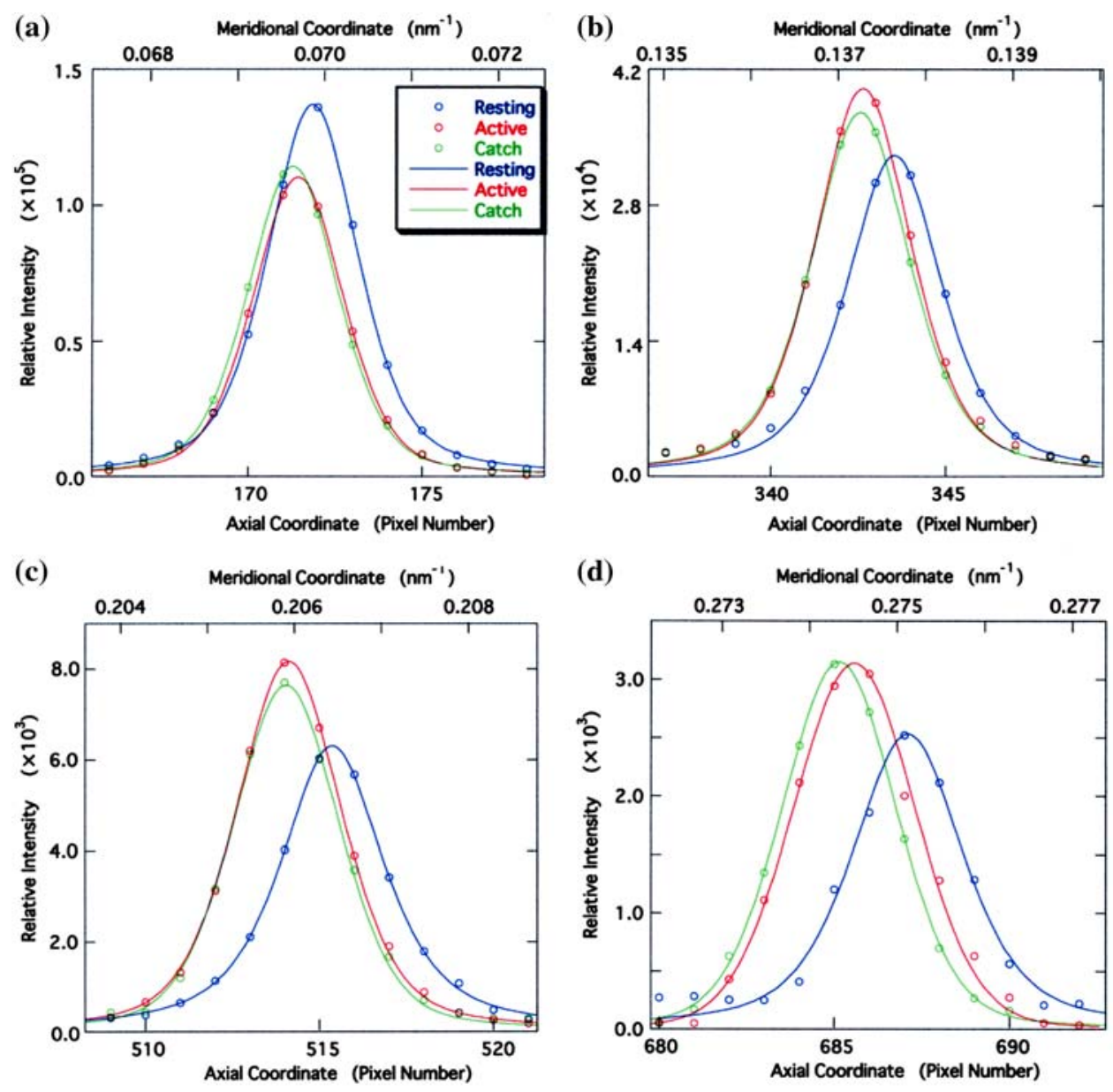

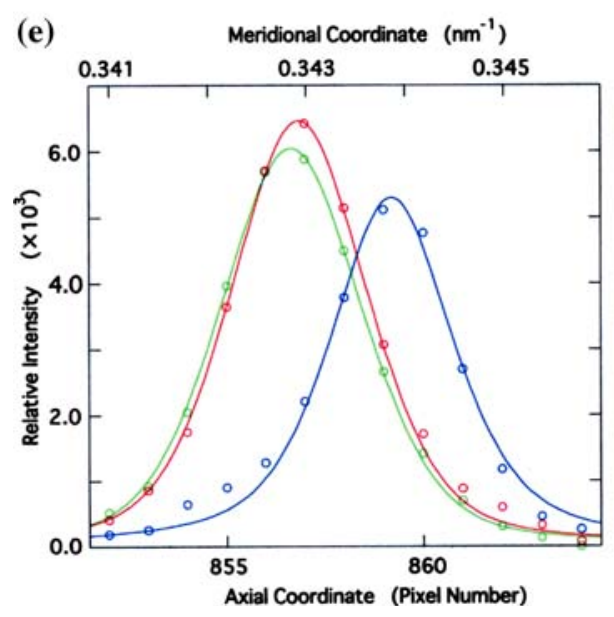

the axial spacings of these reflections actually increased with the transition from the active to the catch state (Fig. 6). Elongations of the axial spacings obtained from the fitting curves of the intensity distributions shown in Fig. 6 are included in Table 3 as those of Specimen (1), with two other examples. In the three specimens, the tension in the active state exceeded $10 \mathrm{~kg} / \mathrm{cm}^{2}$, and tension decay after the end of the stimulation was less than $23 \%$.
The elongation of the axial spacings in the catch state obviously depended on the extent of catch relaxation. If the tension decay after the end of the stimulation was above $30 \%$, the axial spacings were smaller in the catch state than in the active state, even if the tension in the active state was above $10 \mathrm{~kg} / \mathrm{cm}^{2}$. This change occurred in almost the same range where the intensity of the equatorial peak begins to increase appreciably (Lowy and Poulsen 1982). 
Table 3 Increases in the axial spacings of the meridional reflections from the thick filaments in the active and following catch states

\begin{tabular}{|c|c|c|c|c|c|c|}
\hline \multirow[t]{3}{*}{ Spacing } & \multicolumn{6}{|c|}{ Specimen } \\
\hline & \multicolumn{2}{|l|}{ (1) } & \multicolumn{2}{|l|}{ (2) } & \multicolumn{2}{|l|}{ (3) } \\
\hline & Active & Catch & Active & Catch & Active & Catch \\
\hline $14.5 \mathrm{~nm}$ & $0.24 \%$ & 0.33 & 0.29 & 0.32 & 0.48 & 0.53 \\
\hline 7.3 & 0.25 & 0.28 & 0.25 & 0.29 & 0.46 & 0.55 \\
\hline 4.8 & 0.24 & 0.26 & 0.25 & 0.29 & 0.46 & 0.54 \\
\hline 3.6 & 0.22 & 0.29 & 0.22 & 0.27 & 0.46 & 0.54 \\
\hline 2.9 & 0.27 & 0.30 & 0.26 & 0.29 & 0.44 & 0.50 \\
\hline Average & 0.24 & 0.29 & 0.25 & 0.29 & 0.46 & 0.53 \\
\hline SD & 0.018 & 0.026 & 0.025 & 0.018 & 0.014 & 0.019 \\
\hline
\end{tabular}

The three meridional reflections from the thick filaments changed in the integrated intensity in the active and catch states (Figs. 3 and 6). Unlike the spacings, the integrated intensities in the active state partially recovered in the catch state toward those in the resting state (Table 1).

Thin filament layer line reflections

Figures 7 and 8 show the intensity distributions across the 5.9 and $5.1 \mathrm{~nm}$ layer lines from the thin filaments shown in Fig. 1. The $5.9 \mathrm{~nm}$ reflection tends to form an arc with distance from the meridian. This is due to distortion of the thin filament orientation, and is especially pronounced in the resting state. The intensity distributions shown in Fig. 7a were obtained by radial integration in a region close to the meridian where the $5.9 \mathrm{~nm}$ reflection appeared to be relatively straight. The axial spacings of the 5.9 and $5.1 \mathrm{~nm}$ layer lines in the three states were obtained from the maximum points on the fitting curves shown in Fig. 7b-e (Table 4). The spacings increased in the active state and partially recovered in the catch state toward those in the resting state. The elongations of the axial distance between actin molecules in the thin filament, as estimated from the spacing changes in the 5.9 and $5.1 \mathrm{~nm}$ layer lines, were 0.93 and $0.67 \%$ in the active and catch states, respectively.

The intensity distributions shown in Fig. 8a were obtained by radial integration of the intensity in the region that included the whole $5.9 \mathrm{~nm}$ layer line reflection. The intensities of the 5.9 and $5.1 \mathrm{~nm}$ layer lines were obtained by integrating the areas under the fitting curves shown in Fig. 8b (Table 4). As shown previously (Tajima et al. 1994b), the integrated intensity of the $5.9 \mathrm{~nm}$ layer line was weaker in the active state than in the resting state, and partially recovered in the catch state. On the other hand, the integrated intensity of the $5.1 \mathrm{~nm}$ layer line was stronger in the active state than in the resting state, but partially recovered in the catch state also.
The $19.5 \mathrm{~nm}$ layer line reflection, which was undetectable in the resting state, increased in intensity in the active state and weakened in the catch state (Figs. 1a and 9), in agreement with the work of Popp et al. (1991). A comparison of the integrated intensities obtained by integrating the areas under the intensity profiles in Fig. 9 showed that the transition from the active to the catch state reduced the intensity of the $19.5 \mathrm{~nm}$ reflection to $71 \%$.

Rigor-like reflections

It is known that the rigorized ABRM shows layer line reflections from the thin filaments that are decorated with myosin heads according to the symmetry of the actin helix (Lowy and Vibert 1972; Tajima et al. 1999b). In the smallangle X-ray diffraction pattern from the ABRM in the active state, weak reflections similar to the layer line reflections in the rigor pattern appeared under the $5.9 \mathrm{~nm}$ layer line (Fig. 10) (Tajima et al. 1994b, 1999a). Timeresolved small-angle X-ray diffraction showed that the rigor-like reflections increased in intensity with the increase in tension induced by stimulation with either a high $\left(10^{-3} \mathrm{M}\right)$ or low $\left(10^{-5} \mathrm{M}\right)$ concentration of $\mathrm{ACh}$ solution. In the catch state, the rigor-like reflections became very weak although they were still faintly visible. Therefore, the arrangement of the thin and thick filaments in the catch state was different from the rigor structure, in which all the myosin heads attach to the thin filaments to join the thin and thick filaments throughout their overlap region.

\section{Equatorial reflection}

The intensity distribution along the equator was separated into an equatorial peak attributable to the organization of the thin filaments and a smooth background (Fig. 11). The integrated intensity of the equatorial peak was weaker in the active state than in the resting state (Table 5). In the catch state, the integrated intensity increased slightly but was almost equal to that in the active state, in agreement with the work of Lowy and Poulsen (1982).

\section{Discussion}

The small-angle X-ray diffraction patterns from the isometrically contracted ABRM, which developed strong tensions in both the active and catch states, showed elongations of the axial spacings of the meridional reflections from the thick filaments upon the transition from the active to the catch state. This result implies that the thick filaments were more elongated in the catch state than in the active state, probably due to the increase in tension applied 
Fig. 7 Background-subtracted intensity distributions obtained by radial integration within the region $0-0.094 \mathrm{~nm}^{-1}$, where the $5.9 \mathrm{~nm}$ layer line reflection appeared to be relatively straight without an appreciable disorientation effect.

(a) Comparison of the observed intensity distributions among the resting, active, and catch states. The intensity

distributions in the (b) resting, (c) active, and (d) catch states were separated into four peaks: the two peaks of the 5.9 and $5.1 \mathrm{~nm}$ layer lines from the thin filaments, and the two peaks of the off-meridional 6.1 and $5.6 \mathrm{~nm}$ reflections from the thick filaments. Circle: Observed intensity. Solid line: Fitting curve. (e) Comparison of the four separated peaks among the three states
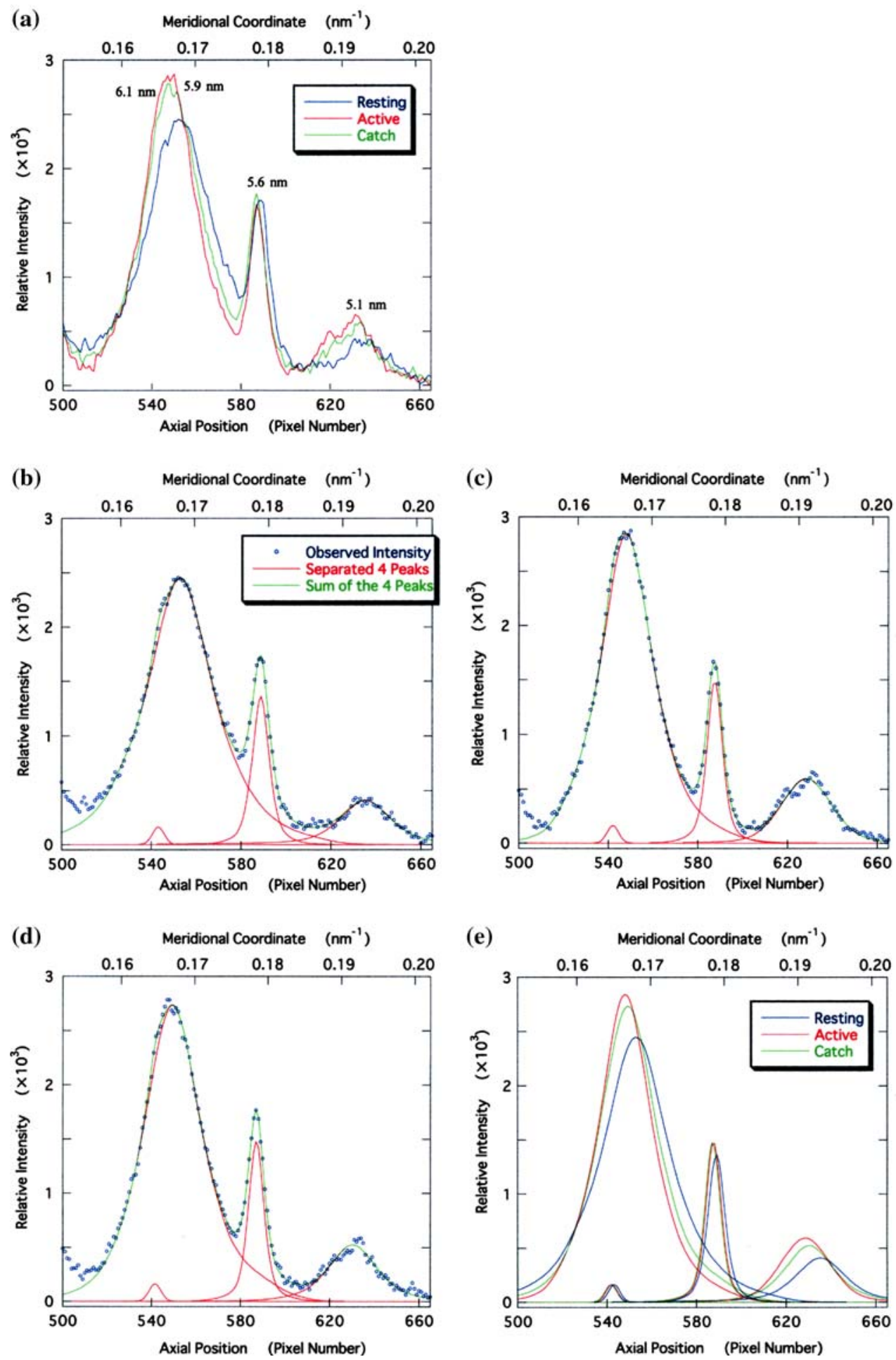

to the thick filaments. Because the intensity of the equatorial peak in the catch state was almost equal to that in the active state, the two states were similar in their distributions of the thin and thick filaments, in accordance with the electron microscopic observation of Bennett and Elliott (1989). The appearance of the $19.5 \mathrm{~nm}$ layer line reflection in the catch state indicated that a great portion of the thin filaments was in the activated structure. The thick filaments in the catch state were elongated probably by the association between thin and thick filaments.

The catch mechanism has been related to the structure of the muscle in two ways. Two main hypotheses, the paramyosin hypothesis (Johnson et al. 1959) and the linkage hypothesis (Lowy and Millman 1963), have been 
Fig. 8 Background-subtracted intensity distributions obtained by radial integration within the region $0-0.210 \mathrm{~nm}^{-1}$, which contained the whole of the $5.9 \mathrm{~nm}$ layer line reflection from the thin filaments shown in Fig. 1a. (a) Comparison of the observed intensity distributions among the resting, active, and catch states. (b) Comparison of the four separated peaks among the three states
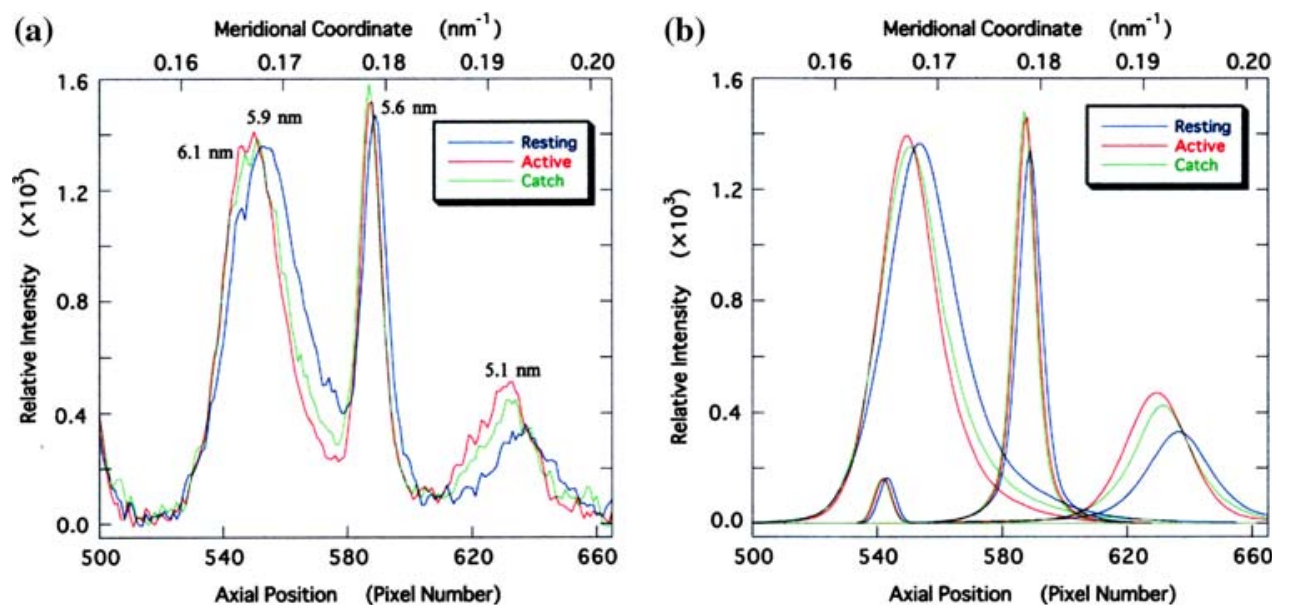

Table 4 Changes in the axial spacings and integrated intensities of the 5.9 and $5.1 \mathrm{~nm}$ layer line reflections from the thin filaments

\begin{tabular}{llll}
\hline State & $\begin{array}{l}\text { Spacing } \\
(\mathrm{nm})\end{array}$ & $\begin{array}{l}\text { Spacing change } \\
(\%)\end{array}$ & $\begin{array}{l}\text { Intensity change } \\
(\%)\end{array}$ \\
\hline $5.9 \mathrm{~nm}$ & & & 0 \\
Resting & 5.945 & 0 & -9.72 \\
Active & 5.989 & 0.73 & -4.18 \\
Catch & 5.977 & 0.54 & \\
$5.1 \mathrm{~nm}$ & & & 0 \\
Resting & 5.170 & 0 & 30.6 \\
Active & 5.227 & 1.11 & 18.6 \\
Catch & 5.211 & 0.79 & 0.6 \\
\hline
\end{tabular}

The axial spacings and the integrated intensities were obtained from the separated peaks shown in Figs. 7e and 8b, respectively

proposed to explain the prolongation of tension in the catch phase. In the linkage hypothesis, catch is related to the persistence of the strongly bound actin-myosin linkages between the thin and thick filaments. Here, the association between the thin and thick filaments is explicitly incorporated into the catch mechanism. However, it has been found that catch is insensitive to myosin inhibitors such as vanadate (Galler et al. 2005; Butler et al. 2006). The catch state is regulated by the c-AMP-dependent phosphorylation of twitchin, which is located on the thick filament (Siegman et al. 1997, 1998). Twitchin phosphorylation and moderate intracellular alkalization, either of which terminates the catch state, do not accelerate myosin head detachment (Andruchova et al. 2005; Hopflinger et al. 2006). Therefore, it is unlikely that catch tension is maintained by actin-myosin linkages. Butler et al. (2006) suggested that catch force is generated by dephosphorylated twitchin, which provides a linkage between the thin and thick filaments, and that myosin regulates the attachment and detachment of twitchin to the thin filament. Recently, Funabara et al. (2007) proposed that the complex

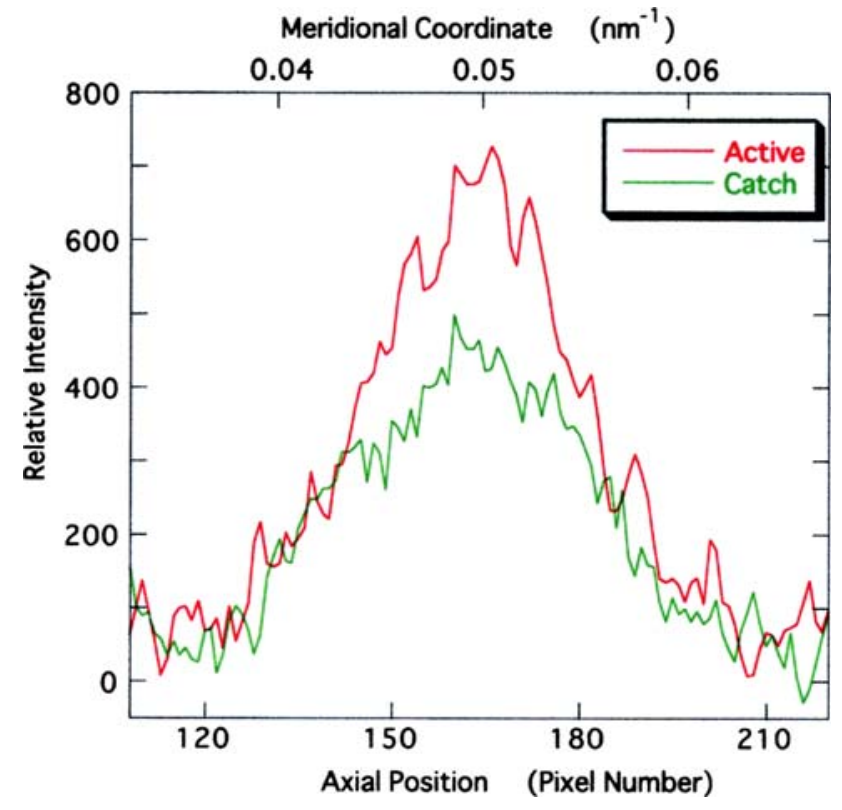

Fig. 9 Background-subtracted intensity distributions of the $19.5 \mathrm{~nm}$ layer line reflection from the thin filaments in the active and catch states. The intensity distributions were obtained by radial integration in the region $0.138-0.304 \mathrm{~nm}^{-1}$, which contained the whole of the $19.5 \mathrm{~nm}$ layer line reflection shown in Fig. 1a

formed with the dephosphorylated D2 site of twichin, Factin and myosin represents a component of the mechanical linkage responsible for catch force maintenance.

According to Cornelius (1982), the catch tension that appears soon after the end of the stimulation decreases exponentially. The tension decay is probably due to a gradual decrease in the number of linkages between the thin and thick filaments. Because these filaments are bound to each other by the remaining linkages, tension decay cannot be brought about by sliding them against each other's full lengths. It is supposed that each thin filament becomes somewhat more extensible or unable to maintain tension at the parts where the linkages become detached 


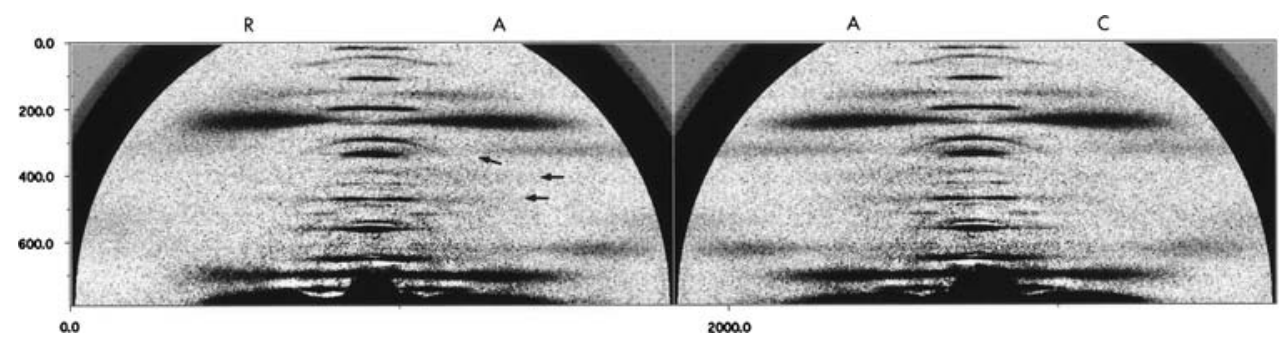

Fig. 10 Background-subtracted small-angle X-ray diffraction patterns shown in Fig. 1, compared between the resting (R), active (A), and catch $(\mathrm{C})$ states. The rigor-like layer line reflections corresponding to the axial spacings, 7.0, 8.6, and $11.1 \mathrm{~nm}$, are indicated by the arrows. The $10.4 \mathrm{~nm}$ off-meridional reflection from the thick filaments showed a relatively strong secondary reflection, which overlapped on the $11.1 \mathrm{~nm}$ rigor-like reflection. The secondary reflection did not change in intensity between the active and catch states
Fig. 11 (a) Intensity distributions of the equatorial reflection axially integrated within the width of the equatorial peak in the resting state, i.e., the largest width among those in the resting, active, and catch states. The chain lines show the backgrounds of the equatorial peaks, which are expressed by polynomials fitted to the intensity on either side of each peak. (b) Intensity distributions of the equatorial peaks obtained by background-subtraction
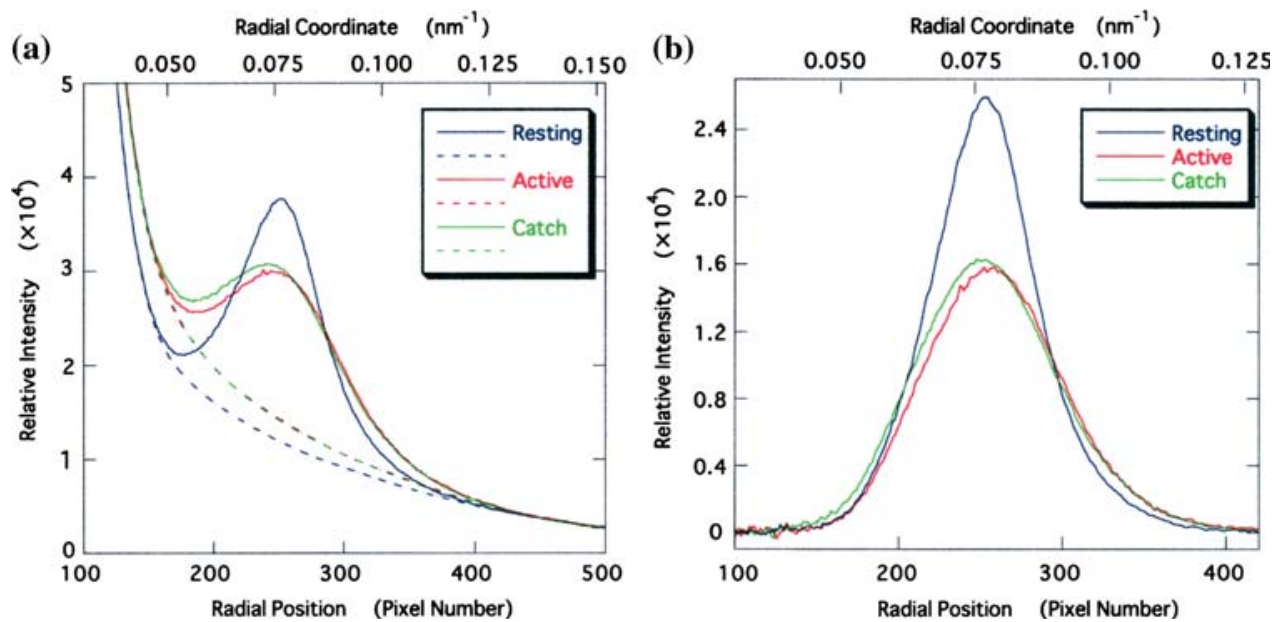

Table 5 Changes in the equatorial peaks in the active and catch states

\begin{tabular}{llll}
\hline State & $\begin{array}{l}\text { Spacing } \\
(\mathrm{nm})\end{array}$ & $\begin{array}{l}\text { Half-width } \\
\left(\times 10-1 \mathrm{~nm}^{-1}\right)\end{array}$ & $\begin{array}{l}\text { Intensity change } \\
(\%)\end{array}$ \\
\hline Resting & 12.98 & 2.23 & 0 \\
Active & 12.83 & 3.01 & -21.3 \\
Catch & 13.07 & 3.02 & -17.1
\end{tabular}

${ }^{a}$ Change in the intensity obtained by integration of the area below the intensity curve shown in Fig. 11b

(Fig. 12a). Accordingly, the tensile stress in the overlap region between the thin and thick filaments will be depressed, and the thick filaments will come to play a more important role than the thin ones in maintaining the tension. The depression of the tensile stress in the overlap region disturbs the equilibrium of the tensile stress between the overlap region and the region that includes the dense body and thin filaments connected to it. To reach a new equilibrium state, the overlap region becomes elongated and the region that includes the dense body contracts (Fig.12b). This may be the reason for the thick filament elongation in the catch state.
A decrease in the number of myosin cross-bridges around the thin filaments in the catch state is suggested by intensity changes of the meridional reflections from the thick filaments. This might cause a decrease in the number of twitchin linkages between the thin and thick filaments. After the extent of catch relaxation exceeds $30 \%$, the thin filaments gradually detach from the thick filaments, and gather to make groups of them. This will reduce the tension applied to the thick filaments.

The paramyosin hypothesis suggests that the tension developed by the actin-myosin linkages in the active state is preserved in the catch state by the interconnecting paramyosin-containing thick filaments. Actually, thick filament interconnections are observed in the catch state abundantly in electron microscopic studies of the freezesubstituted ABRM, and it is assumed that the interconnections are formed by twitchin (Takahashi et al. 2003). The paramyosin hypothesis is favored for its possibility of explaining the very high stiffness of the muscle in the catch state (Johnson and Twarog 1960; Sugi et al. 1999). However, the paramyosin hypothesis faces a problem regarding tension propagation. It has been shown that the thin filaments are connected to the cell membrane by the dense bodies attached to it (Twarog et al. 1973; Sobieszek 1973). 


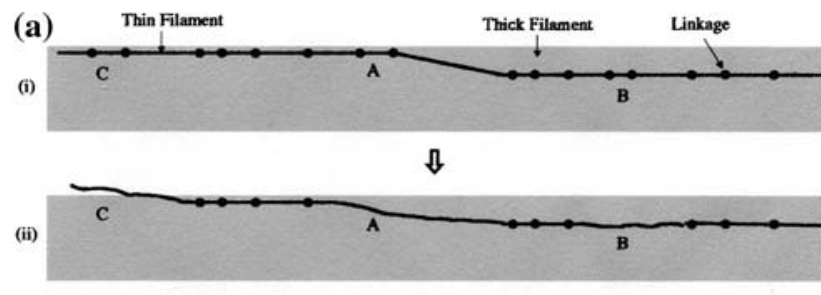

(b)

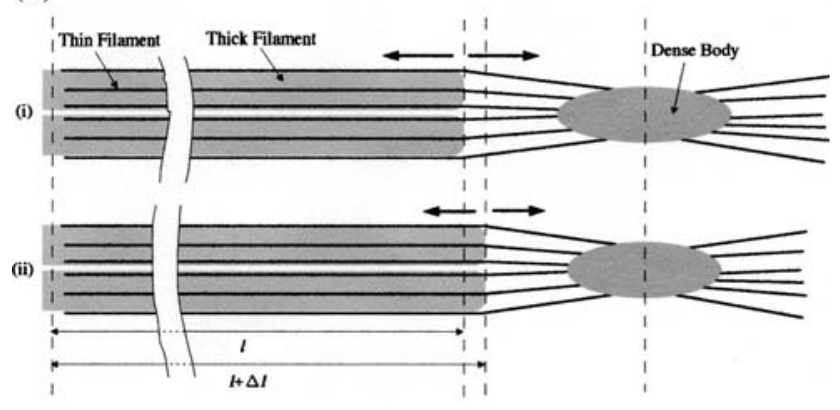

Fig. 12 Illustration of the thick filament elongation in the catch state. (a) Schematic diagram of the conformational change of the thin filament due to dissociation of a part of the linkages between the thin and thick filaments. (i) Before dissociation. (ii) After dissociation. Part A: Because the thin filament is not parallel to the axis of the thick filament, it becomes loose after dissociation of the linkages. The thin filament may also change its direction between the adjacent thick filaments by changing the binding position from one thick filament to another. According to the electron microscopic studies (Bennett and Elliott 1989), the thin filaments are not likely parallel to the thick filaments. Part B: Although the thin filament is parallel to the axis of the thick filament, it may become loose due to the disappearance of a strain, such as the torsion brought about by connection with the thick filament. Part C: A part of the thin filament detaches from the thick filament and does not play a role in maintaining the catch tension. (b) Equilibrium of the tensile stress between the overlap region and the region including a dense body and thin filaments at the beginning of catch (i) is lost after dissociation of a part of the linkages between the thin and thick filaments, and reaches a new equilibrium state (ii). The changes in tensile stresses are schematically shown by arrows. $l$ and $\Delta$ $l$ are the half-length of the thick filament and its elongation, respectively

These dense bodies appear to serve as cell junctions to propagate the tension of the thin filaments from cell to cell. On the other hand, no connection between the thick filaments and the cell membrane has been found. It is uncertain whether or not the thick filaments, by themselves, develop tension that propagates along the whole muscle.

In order to solve this problem, Galler et al. (2005) assumed connections of the thick filaments with the thin filaments and/or with the dense bodies by twitchin besides interconnections between the thick filaments. From our studies of the small-angle X-ray diffraction patterns, connections with the thin filaments are probable. The connections with the dense bodies will be advantageous for explaining the residual stiffness that exists after relaxation of the catch tension (Sugi et al. 1999). However, it seems difficult for such connections to form because of the small diameter of the dense body. We strongly feel that more electron microscopic studies of the longitudinal section of the ABRM in the active and catch states are needed to clarify structural changes at high levels of the contracting system; for example, changes in the distribution and conformation of the thin and thick filaments, or configurational changes in the dense body.

In our previous work on medium-angle X-ray diffraction patterns from the ABRM, the axial spacing of the $1.038 \mathrm{~nm}$ meridional reflection from the thick filaments was smaller in the catch state than in the active state, although both the active and catch tensions seemed sufficiently high (Tajima et al. 1994a). However, the elongation of the thin filaments in the active state, $0.48 \%$, corresponding to the spacing change of the $0.922 \mathrm{~nm}$ meridional reflection from the thin filaments, is smaller than the elongation, $0.93 \%$, estimated in this work from the X-ray pattern shown in Fig. 1. The tension was probably overestimated because of the difficulty of measuring the cross-sectional area of the specimen. However, in this case too, the recovery of elongation by the change from the active to the catch state was smaller in the thick filaments than in the thin ones. There is a possibility that strong tension was applied to the thick filament in the catch state.

Our skill in sample preparation and our X-ray diffraction technique have progressed. The X-ray patterns shown in this work are of much higher quality than the patterns we showed in 1994.

Acknowledgments We wish to express our sincere thanks to Prof. K. Wakabayashi (Osaka Univ.) and to Dr. Y. Sugimoto, Dr. Y. Takezawa, and Prof. Y. Amemiya (Univ. of Tokyo) for their help with our experiments at the Photon Factory. One of the authors is also very grateful to Prof. T. Miyahara (Tokyo Metropolitan Univ.) for providing a position in which she could continue this work. This work has been performed under the approval of the Photon Factory Program Advisory Committee (Proposal Nos. 94G080 and 2000G154).

Open Access This article is distributed under the terms of the Creative Commons Attribution Noncommercial License which permits any noncommercial use, distribution, and reproduction in any medium, provided the original author(s) and source are credited.

\section{References}

Andruchova O, Hopflinger MC, Andruchov O, Galler S (2005) No effect of twitchin phosphorylation on the rate of myosin head detachment in molluscan catch muscle: are myosin heads involved in the catch state? Pflugers Arch 450:326-334

Bennett PM, Elliott A (1989) The 'catch' mechanism in molluscan muscle: an electron microscopy study of freeze-substituted anterior byssus retractor muscle of Mytilus edulis. J Muscle Res Cell Motil 10:297-311. doi:10.1007/BF01758426

Butler TM, Mooers SU, Siegman MJ (2006) Catch force links and the low to high force transition of myosin. Biophys J 90:3193-3202. doi:10.1529/biophysj.105.077453

Cornelius F (1982) Tonic contraction and control of relaxation in a chemically skinned molluscan smooth muscle. J Gen Physiol 79:821-834. doi:10.1085/jgp.79.5.821 
Funabara D, Hamamoto C, Yamamoto K, Inoue A, Ueda M, Osawa R et al (2007) Unphosphorylated twitchin forms a complex with actin and myosin that may contribute to tension maintenance in catch. J Exp Biol 210:4399-4410. doi:10.1242/jeb.008722

Galler S, Hopflinger MC, Andruchov O, Andruchova O, Grassberger $\mathrm{H}$ (2005) Effects of vanadate, phosphate and 2, 3-butanedione monoxime (BDM) on skinned molluscan catch muscle. Pflugers Arch 449:372-383

Hopflinger MC, Andruchova O, Andruchov O, Grassberger H, Galler $\mathrm{S}$ (2006) Effect of $\mathrm{pH}$ on the rate of myosin head detachment in molluscan catch muscle: are myosin heads involved in the catch state? J Exp Biol 209:668-676. doi:10.1242/jeb.02033

Johnson WH, Twarog BM (1960) The basis for prolonged contractions in molluscan muscles. J Gen Physiol 43:941-960. doi: 10.1085/jgp.43.5.941

Johnson WH, Kahn JS, Szent-Gyorgyi AG (1959) Paramyosin and contraction of 'catch muscles'. Science 130:160-161. doi: 10.1126/science. 130.3368 .160

Lehman W, Kendrick-Jones J, Szent-Gyorgyi AG (1972) Myosinlinked regulatory systems: comparative studies. Cold Spring Harb Symp Quant Biol 37:319-330

Lowy J, Millman BM (1963) The contractile mechanism of the anterior byssus retractor muscle of Mytilus edulis. Phil Trans R Soc B 246:105-148. doi:10.1098/rstb.1963.0003

Lowy J, Poulsen FR (1982) Time-resolved X-ray diffraction studies of the structural behaviour of myosin heads in a living contracting unstriated muscle. Nature 299:308-312. doi:10.1038/ 299308a0

Lowy J, Poulsen FR (1987) X-ray study of myosin heads in contracting frog skeletal muscle. J Mol Biol 194:595-600. doi: 10.1016/0022-2836(87)90236-1

Lowy J, Vibert PJ (1972) Studies of the low-angle X-ray pattern of a molluscan smooth muscle during tonic contraction and rigor. Cold Spring Harb Symp Quant Biol 37:353-359

Popp D, Maeda Y, Stewart AAE, Holmes KC (1991) X-ray diffraction studies on muscle regulation. Adv Biophys 27:89103. doi:10.1016/0065-227X(91)90010-B

Poulsen FR, Lowy J (1983) Small-angle X-ray scattering from myosin heads in relaxed and rigor frog skeletal muscles. Nature 303:146-152. doi:10.1038/303146a0

Siegman MJ, Funabara D, Kinoshita S, Watabe S, Hartshorne DJ, Butler TM (1998) Phosphorylation of a twitchin-related protein controls catch and calcium sensitivity of force production in invertebrate smooth muscle. Proc Natl Acad Sci USA 95:53835388. doi:10.1073/pnas.95.9.5383
Siegman MJ, Mooers SU, Li C, Narayan S, Trinkle-Mulcahy L, Watabe $S$ et al (1997) Phosphorylation of a high molecular weight (approximately $600 \mathrm{kDa}$ ) protein regulates catch in invertebrate smooth muscle. J Muscle Res Cell Motil 18:655670. doi:10.1023/A:1018683823020

Sobieszek A (1973) The fine structure of the contractile apparatus of the anterior byssus retractor muscle of Mytilus edulis. J Ultrastruct Res 43:313-343. doi:10.1016/S0022-5320(73)80041-3

Sugi H, Iwamoto H, Shimo M, Shirakawa I (1999) Evidence for loadbearing structures specialized for the catch state in Mytilus smooth muscle. Comp Biochem Physiol 122A:347-353

Tajima Y, Makino K, Wakabayashi K, Amemiya Y (2001) X-ray diffraction studies of a molluscan smooth muscle in the catch state. Photon Factory Activity Report 2000 \#18 part B:246

Tajima Y, Makino K, Hanyuu T, Wakabayashi K, Amemiya Y (1994a) X-ray evidence for the elongation of thin and thick filaments during isometric contraction of a molluscan smooth muscle. J Muscle Res Cell Motil 15:659-671. doi:10.1007/ BF00121073

Tajima Y, Wakabayashi K, Amemiya Y (1994b) X-ray diffraction studies of the thin filaments in a contracting molluscan smooth muscle. In: Chance B, Deisenhofer J, Ebashi S, Goodhead DT, Helliwell JR, Huxley HE, Iizuka T, Kirz J, Mitsui T, Rubenstein E, Sakabe N, Sasaki T, Schmahl G, Stuhrmann HB, Wutrich K, Zakkai G (eds) Synchrotron Radiation in the Biosciences. Oxford University Press, pp 509-518

Tajima Y, Makino K, Hanyuu T, Wakabayashi K, Amemiya Y (1999a) The overlap between the thin- and thick-filament reflections in the small-angle X-ray diffraction pattern from a molluscan smooth muscle. J Synchrotron Radiat 6:93-100. doi: 10.1107/S0909049598015544

Tajima Y, Tajima M, Iwasaki H, Makino K, Wakabayashi K, Amemiya Y (1999b) Small-angle X-ray diffraction studies of a molluscan smooth muscle in the rigor state. Photon Factory Activity Report 1998 \#16 part B:266

Takahashi I, Shimada M, Akimoto T, Kishi T, Sugi H (2003) Electron microscopic evidence for the thick filament interconnections associated with the catch state in the anterior byssal retractor muscle of Mytilus edulis. Comp Biochem Physiol 134A: $115-120$

Twarog BM, Dewey MM, Hidaka T (1973) The structure of Mytilus smooth muscle and the electrical constants of the resting muscle. J Gen Physiol 61:207-221. doi:10.1085/jgp.61.2.207 